in several papers $(1878-80)$ with the gas spectra in a continuation of Plücker's earlier work. Warren de la Rue, Hugo Müller and Spottiswoode described, in 1875, experiments with a new chloride of silver bsttery of 1,080 cells, which in subsequent work increased to the truly monumental number of 14,400 . This was demonstrated at a Friday Evening lecture at the Royal Institution on January 21, 1881, by Warren de la Rue.

In 1886, Goldstein, whose name has already been mentioned, discovered the 'Canalstrahlen' or positive rays which were later investigated by Wien and J. J. Thomson.

With the increase of knowledge of cathode rays came the inquiry into their nature. There were two views: one assumed the cathode rays to be corpuscular, the other regarded them as disturbances in the ether. On the whole, British scientific men adhered to the former view. Crookes invented the term ' $a$ fourth radiant state of matter'. On the Continent, a number of investigators favoured the ether view. There were, however, exceptions. Riecke published in 1881 a short note on the path of an electrically charged particle with finite mass in a magnetic field, and pointed out that Hittorf's experiments could be interpreted by assuming a cathode ray to be a stream of such particles moving with uniform velocity. Similar calculations were made in 1876 by Stokes, and these were published in an article by Spottiswoode. These calculations, however, dealt with the deformation of a flexible current filament (spark) and no mention is made of particles.

The idea of atomistic electricity was first announced by Johnstone Stoney ${ }^{18}$ in a lecture at the Belfast peeting of the British Association in 1874 and was later published by him in 1881 in the Philosophical Magazine. At the same time, Helmholtz brought forward the same idea in a lecture in Germany. Johnstone Stoney gave the atom of negative electricity the now familiar name 'electron'.

The general theory of discharge of electricity in gases, incorporating both the positive and negative atomic charges, was first given by A. Schuster ${ }^{10}$, who made a brilliant analysis of the whole problem of the electrical conductivity of gases. His three papers mark the beginning of a new development in this field of research.

We are now rapidly moving toward Röntgen's discovery, but there are two more events to record. In 1892, H. Hertz ${ }^{20}$, who previously had made the great discovery of electromagnetic waves, published \& paper in which he showed that cathode rays can pass through thin metal foils. This important fact must have influenced Prof. Ph. Lenard ${ }^{21}$, who thus perceived the possibility of investigating cathode rays escaping through a thin aluminium window in the discharge tube. Lenard's famous tube and the equally remarkable experiments with cathode rays were described by him in his well-known paper. After this, the stage was set for Röntgen's discovery in 1895 .

Röntgen sent his first communication to the Würzburger Physikalische-Medizinische Gesellschaft, a local scientific society, which published it towards the end of the year 1895. The paper bears the title, "Uber eine neue Art von Strahlen". A few months later, in 1896, he made a second communication and these two, together with a third, appeared in the Annalen der Physik 22 in 1898.

The new rays, or $\mathrm{X}$-rays as the discoverer called them, were investigated with extreme thoroughness by Röntgen, and although a large number of investi- gations were made and reported in 1896 by his contemporaries all over the world, no essentially new facts appeared for several years. This holds in particular for his discovery of the ionization produced by $\mathbf{X}$-rays in gases and solids.

Bearing in mind the fact that $\mathrm{X}$-rays had been produced in many previous investigations, as has already been shown, the question arises why Röntgen succeeded when others failed. At that time Röntgen could not have predicted the existence of X-rays on theoretical grounds. In his communications he makes no claims to prediction, and simply states what he had found. He does not say why he chose the particular combination of apparatus that gave him his results. Zehnder, a contemporary of Röntgen, who was for many years his assistant, though not at the time when $\mathbf{X}$-rays wore discovered, gives a very plausible explanation in his biography of Röntgen, "Röntgens Briefe an Zehnder" 23. From this we gather that in 1894 Röntgen had required one of Lenard's tubes and that he was much impressed by its performance. According to Zehnder, Röntgen most likely tried to see whether or not some of Lenard's rays might escape into the open from an ordinary Hittorf vacuum tube, and in carrying out the experiment be discovered the new rays.

The discovery was therefore to a great extent a stroke of good luck that any of Röntgen's predecessors since Gassiot might have had. Röntgen's great achievement lies in his thorough investigation of the properties of the new rays. The element of luck inherent in any discovery only enhances the fascination attached to the study of the history of an idea and of a tool, that from their embryonic state in Faraday's time furnished now and important knowledge after a long period of development.

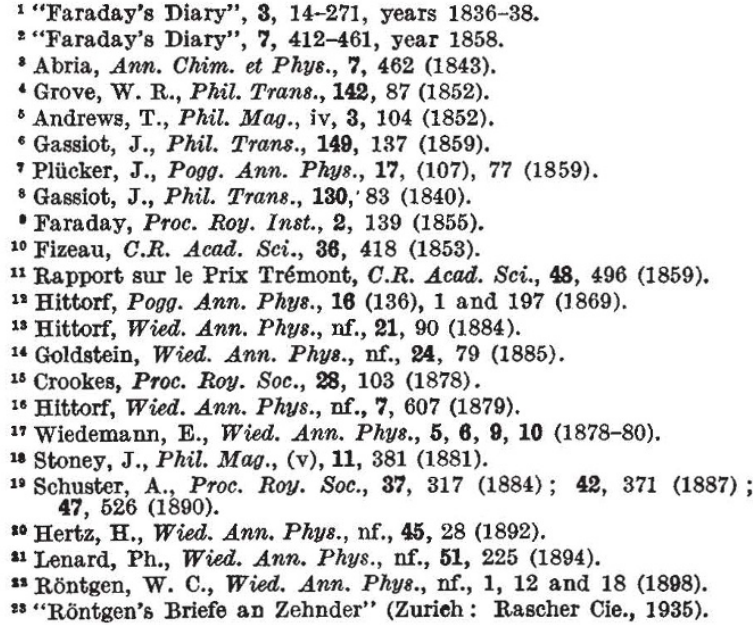

\section{PROGRESS OF X-RAY ANALYSIS OF ORGANIC AND FIBRE STRUCTURES}

\author{
By Prof. W. T. ASTBURY, F.R.S. \\ University of Leeds
}

$F_{\mathrm{H}}^{\mathrm{o}}$ OR the mornings of November 9 and 10 of the Röntgen Celebrations, the Institute of Physics arranged two sessions held at the Royal Institution and devoted to a series of short papers on pro- 
gress and prospects in X-ray structure analysis. J. M. Robertson and Dorothy Crowfoot (Mrs. Hodgkin) spoke on organic crystals, and W. T. Astbury on fibre structures; and since their remarks link up together, it is convenient to summarize them in the order just given.

(1) Organic structures represent the greatest single field for the application of the methods of X-ray erystal analysis. Progress was slow at first because the structures are nearly always complicated, with many similar atoms occupying general positions in the unit cell; but now that the initial difficulties have been overcome, many accurate solutions have been worked out by the methods of successive approximation by means of Fourier series. Striking advances of a purely crystallographic kind are still by no means easy, however, for the unusual reason that the organic chemist has already made such tremendous progress that it is quite a problem to provide him with information with which he is not already familiar !

Broadly speaking, the field open to X-ray analysis falls into three principal domains. First, there are the numerous complex compounds that lie on or near the boundary of the subject and the structure of which is still unknown to the chemist. Progress here is possible only in close collaboration with the latter, and then it not infrequently happens that a few simple X-ray measurements give information about the general shape of the molecule that eliminates a number of possibilities at once. Later, special groupings and configurations may be tested, but this is mostly very difficult unless heavy atoms can be placed in the molecule at predetermined points, or isomorphous series can be prepared. Notable contributions of this sort have been made by Bernal in his early work on the sterols, and by Miss Crowfoot in recent more detailed work on similar compounds.

Secondly, there are the compounds, more numerous than might be thought, the structures of which are known to the chemist in broad outline but not in detail. If a compound belongs to the aromatic series, the chemical structural formula now enables us to tell the spatial positions of its atoms with a good deal of precision; but if it is fully hydrogenated, then the situation may be very different : numerous isomers are possible that from the chemical point of view are hard to identify. For example, our know. ledge of the configurations of even the simple cyclohexane derivatives is far from complete, and the precise structures of the various isomers of that now very important substance, benzene hexachloride, are largely unknown. In directions such as these there is great scope for X-ray analysis, and a considerable amount of work is in progress in Glasgow. Generally speaking, however, the problems involved are proving to be very difficult.

Thirdly, there are all the organic compounds the structures of which are now well known to the chemist in the sense that he knows the relative spatial positions of all the atoms, together with something about their types of bonding. X-ray analysis can add much to this kind of information by translating it into absolute terms, rather in the way the new X-ray crystallography has transformed the axial ratios of the old classical crystallography. The advances actually made have been very great indeed, and now form the basis of a large part of modern theoretical chemistry. As two examples may be mentioned the structures of the aromatic hydrocarbon, coronene, and of certain carboxylic acids. The solution of the coronene structure is of special interest because it is the first case of a purely aromatic condensed-ring hydrocarbon where different bond-lengths have been detected in different parts of the molecule-a state of affairs that can be predicted by theoretical calculations based on the method of molecular orbitals (Coulson). The crystal structure of oxalic acid dihydrate is interesting because it contains one of the shortest known hydrogen bridges between oxygen atoms $(2 \cdot 52 \mathrm{~A}$.) and gives an abnormally large spacing expansion when deuterium is substituted for hydrogen. Until recently this was an isolated case, but the acetylene carboxylic acids have now been shown to behave similarly. Work on these structures is at present in progress in Glasgow.

(2) The problem of solving the structure of a complex organic crystal is effectively that of elucidating the structure of the molecule under investigation. Even the earliest example, that of hexamethylene tetramine in 1923, proved able to distinguish between various likely chemical formulæ; but as the number of possible atomic arrangements increases, the character of the problem changes, and solutions have been sought through the use of Fourier methods applied to isomorphous structures differing in one atomic constituent, or to crystals containing one very heavy atom. Either approach provides information on the phase angles of certain X-ray reflexions, that may be complete as in the case of phthalocyanine (Robertson), or only partial as with $\alpha$-chitosamine hydrochloride and hydrobromide (Cox and Jeffrey) or cholesteryl iodide (Carlisle and Crowfoot). The advantages and imperfections of the method may be illustrated by the analysis of one modification (B) of cholesteryl iodide. Here the heavy atom, iodine, gave the phases necessary for the complete determination of one Fourier projection ; and in this projection, in the light of earlier X-ray and chemical knowledge, it was easy to pick out the outlines of the sterol skeleton. In three dimensions the phase angles are indeterminate. There is little difficulty in separating the effects due to the two molecules in the unit cell, but the placing of the atoms within one molecule is less unambiguous and required some trial-and-error work. The final solution does provide evidence on certain points of sterol stereochemistry with regard to which chemical evidence is either absent or conflicting.

One might ask whether the sterol skeleton would have been recognized in the Fourier pattern if its general form had not been known already. In cholesteryl iodide probably it would; but on the other hand, recent very practical experiences show that it is quite possible to have a correct, or nearly correct, Fourier projection without recognizing in the least which peaks represent which of the known atoms present. In such cases it is a good plan in the early stages to examine more than one-not isomorphous-crystal structure containing the molecule under investigation, while in the later stages one's protection lies in the fact that the final solution must reveal discrete, reasonably well-formed atoms, and the right number of atoms at that. Actually, in this region it is noticeable that it becomes less easy to fix precisely the atomic positions (presumably because they are less precisely fixed in space themselves), and this is evident from the falling-off of the intensity of reflexion at small spacings. A beautiful illustration of the effect is found in Jeffrey's 
analysis of geranylamine hydrochloride : the farther away the carbon atoms are from the ionic centre, the more the spreading of the electron-density contours.

Lastly, the placing of the many atoms in such structures requires very heavy calculations. In the early stages of the analysis the difficulties can be met admirably by the use of the 'fly's eye', or by the Bragg and Lipson structure-factor graphs; but in the later stages it does seem that some professional computing assistance or new developments in machines are called for.

Though it would be rash to set an upper limit to the complexity of structure that might be handled by these methods, it must be confessed that the present outlook with regard to detailed solutions of protein crystals is not very hopeful. They give photographs directly comparable with those of simpler crystals, but in the case of the tobacco necrosis virus, for example, the triclinic unit cell (the largest yet measured) has dimensions $179 \times$ $219 \times 243 \mathrm{~A}$. and contains molecules not far from $200 \mathrm{~A}$. across. One can estimate that out to the observed spacing limit of $2.8 \mathrm{~A}$., one might measure something like a million reflexions; and even with crystals of 'small' proteins one can easily measure five or ten thousand reflexions! The numbers of atoms involved are out of all proportion to the twenty-nine of cholesteryl iodide-literally thousands -and no heavy atom known would control the phase angle. Protein crystals have, however, one property that can assist in their investigation-that of containing large and variable amounts of liquid of crystallization. Frem vector maps given by crystals in various states of hydration, it is possible to show that the molecules are essentially rigid entities, and with some crystals it is possible to obtain also some limited information on the phase angles of reflexions. In this way Perutz has shown that the hæmoglobin probably has a four-layer structure. But even the thousands of available reflexions do not take us down to the limit necessary to distinguish discrete atoms, and it is clear that great caution will have to be exercised in order to make sure of any interpretation in terms of exact chemical arrangement. At one extreme far more work is needed in the region between compounds already analysed and the proteins; while at the other we may hope that the lower limits of resolution of the electron microscope may be extended to check the accuracy with which we can deduce the grosser structure of the protein molecule.

(3) The X-ray study of fibres-natural, artificial, and synthetic-has to do mostly with the structure and states of aggregation of giant molecules about which little precise is known, and among which progress is possible only in close collaboration with other physico-chemical methods of approach. Indeed, since the beginning of the century, the various techniques of the science of high polymers have grown up together, and though it is fair to say that since the 1920's X-rays have played a leading part, giving time and again just those geometrical and dimensional clues needed to co-ordinate the accumulated facts, it still remains that collaboration is essential if ever we are to cope successfully with the multitude of unresolved details. There is scarcely a more worthwhile task, however, if only for the sake of molecular biology, where perhaps more than anywhere else the great future of X-ray analysis lies. To say this is not to disparage technology either, for textiles and plastics too now draw their basic inspiration from the lessons of the natural macromolecules, and the greatest field in present-day chemistry, whether biological or industrial, forms in reality but a single subject, the science of chainmolecules.

It is convenient to subdivide this field into four principal and interrelated sections : polysaccharides, proteins, polynucleotides, and plastics. Cellulose is the polysaccharide-in-chief, and it has been a constant object of interest to the $\mathrm{X}$-ray analyst ever since the close of the First World War. The chemical advances made by Haworth and his school combined with the X-ray interpretation first given by Sponsler and Dore soon led to further important developments at the hands of Meyer and Mark and their co-workers, and altogether, since those days, it is beyond question that the contribution of X-ray methods to our knowledge of cellulose and its derivatives has been invaluable. For a number of years the actual detailed shape of the molecular chain inferred from the X-ray patterns was almost certainly incorrect; but more recent considerations, especially those based on a comparative $\mathrm{X}$-ray study of the new configuration discovered in alginic acid, the polysaccharide from seaweed, supported by the results of Cox and his collaborators working on sugars, have very probably put that right. We are left now with the extremely helpful conclusion that the interatomic distances and interbond angles used in the structure of the polysaccharides are to a first approximation the same as those already found in simpler aliphatic molecules. It is still not possible to prove exactly where each atom is in the cellulose structure-in spite of great improvements in the X-ray photographs the diffraction data are really not sufficient for an exhaustive analysis-but models can now be constructed that must be quite near the truth.

The protein fibres (or more correctly the fibrous proteins), for all their great variety, have yielded to X-rays the somewhat unexpected result that they fall for the most part into only two main configur. ational families, the keratin-myosin-fibrinogen group and the collagen group, distinguished chiefly by the long-range elasticity of the former. In spite of considerable variations in chemical constitution within both groups, these two master plans are preserved throughout. In the keratin-myosinfibrinogen group the unit of structure is a polypeptide grid the normal equilibrium configuration of which is buckled; that is, the main chains are thrown into a series of folds that lie in planes transverse to the cross-linkages; and the basis of the long-range elasticity is the capacity to unfold and refold. The essential structural problem is the nature of the intramolecular fold that characterizes the group; and accumulated evidence now points to a simple system of close packing of the side-chains, with the polars on one side of the main chain and the non. polars on the other. Such a plan is sufficient to explain the observed striking unity amid chemical diversity, and in particular it offers against its proper molecular background the most satisfying general interpretation to date of muscular activity. The plan of the keratin-myosin-fibrinogen group is one of the fundamental ideas in vital architecture : it represents a power of the cell of synthesizing elastic fibrous protein of a standard molecular form, yet the details of which can be adapted to as many ends as the processes of differentiation demand. 
By a generalization of the idea of chain folding first derived from the X-ray study of the fibrous proteins, we arrive at the concept of the corpuscular proteins, and then at the interpretation of denaturation as a process of disorganization whereby the peptide chains are liberated from their specific configurations ; and it has in fact been demonstrated conclusively by $\mathrm{X}$-ray methods (leading to actual industrial processes) that artificial fibrous proteins can be spun, after denaturation, from many corpuscular proteins. By an extension of the idea of close packing of the side-chains, we arrive at the concept of the protein lamina, probably again usually with all or most of the polars on one side and the nonpolars on the other ; and the next step is the building of combinations of two or more laminæ, sometimes alone and sometimes sandwiched with accessory groupings, such as lipoid laminæ. It was an early deduction (1936) from X-ray fibre and monolayer data that the egg albumin molecule consists probably of four superposed laminxe of folded polypeptide chains, and recent physico-chemical results by Lundgren and Palmer strongly support that suggestion. Also, the X-ray findings of Perutz and Boyes-Watson now point to a similar conclusion for the structure of the hæmoglobin molecule.

The full pattern along the molecular chains in both the keratin-myosin-fibrinogen group and the collagen group is on a giant scale as molecules go, so great as to fall within the range of the electron microscope, as has been shown by the recent very beautiful experiments of F. O. Schmitt and his team. Even in unstained collagen fibrils, they have demonstrated a system of light and dark bands corresponding to the $640 \mathrm{~A}$. X-ray fibre period found independently by Bear and Kratky, while in fibrils from certain clam muscles, after treatment with phospho-tungstic acid, they have succeeded in revealing more detail still-a most intriguing arrangement of stainable regions forming a systematically distorted hexagonal net. The impression one gets from combined X-ray and electron microscope studies is of 'patterns within patterns', of successive levels of organization proceeding from the basic plans revealed in large-angle X-ray photographs up to the relatively enormous fibrils, and thence to combinations of fibrils. In this progressive building. up process and the transition from one level to the next, we possibly have to do with small proportions of non-protein components, for example, nucleotides and carbohydrates ; and the suggestion is that it is now necessary to estimate such small-proportion components with much greater precision, to see if they are indeed in definite and structurally significant stoichiometric rolation to the remainder.

The X-ray study of the polynucleotides, like the chemistry of these all-important structures, is as yet practically in its infancy. An encouraging start has been made with sodium thymonucleate, the diffraction pattern of which indicates a columnar piling of flattish nucleotides spaced at almost exactly the same distance as the separation of successive side-chains in an extended polypeptide, and similar features are suggested by preliminary photographs of nucleic acids of the ribose type; but otherwise all this great world remains to be conquered. The proffered reward is tremendous, for, whatever else is in doubt, it seems clear enough now that the innermost secrets of biosynthesis rest above all with the proteins and the nucleic acids.

\section{X-RAY DIFFRACTION IN INORGANIC CHEMISTRY, METALLURGY AND MINERALOGY}

\author{
By DR. H. LIPSON*
}

\section{Chemistry of the Solid State}

$\mathrm{T}$ HE discovery by Friedrich, Knipping and Laue of the diffraction of X-rays by crystals opened up now vistas to those interested in the structure of matter. It was now possible to determine the environment of individual atoms, and this inevitably led to a new and lively interest in the properties of matter, the further study of which had seemed to be opposed by an impassable barrier. Moreover, a new status was given to crystallography. No longer was it to be regarded as a subject of limited interest; all men of science had to have a sound knowledge of crystallographic principles and the ability to apply them to their special problems.

Inorganic chemistry, in particular, was revolutionized by the new concepts that emerged from the determinations of the first crystal structures. Rock salt-the first crystal to be analysed-provided a striking example. It was known, of course, that it dissociates into ions of sodium and chlorine when it dissolves in water; but it was supposed that in the solid state the ions were associated in pairs to form molecules of $\mathrm{NaCl}$; this was the readiest way of accounting for the equality of numbers of sodium and chlorine atoms. Barlow, in 1883, had suggested the true crystal structure; but as his suggestion was not supported by direct experimental evidence it had not reached the general consciousness of chemists. X-ray diffraction showed that Barlow's structure was correct and that there were therefore no molecules of $\mathrm{NaCl}$, but only regular arrangements of chlorine and sodium atoms in which each atom was surrounded by six of the other sort. The equality of numbers of the atoms then appeared to be a result of the equality of their chemical valencies. In this way the beginnings of a new chemistry-crystal chemistry-arose.

This subject may be called the chemistry of the solid state as distinct from the chemistry of the liquid state, which was, and still is, the chemist's main interest. As methods for determining crystal structures improved, so more and more chemical compounds were analysed and provided data for the theoretical chemist. Not only was it possible to determine bond-lengths and inter-bond angles, but also to study ions and infinite complexes that could not exist in the liquid state.

Hydrated inorganic compounds provide a good example of the fruits of this study. The structures of salts with four or six molecules of water could be adequately explained on the basis of Werner's co-ordination theory, the water molecules forming simple regular arrangements about the cation; but what of compounds such as $3 \mathrm{CdSO}_{4} \cdot 8 \mathrm{H}_{2} \mathrm{O}$ or $\mathrm{H}_{3} \mathrm{PW}_{12} \mathrm{O}_{40} .29 \mathrm{H}_{2} \mathrm{O}$ ? The determination of the structures of compounds such as these showed that, in addition to providing a regular grouping around the cation, the water molecules because of their polar

* A review based upon papers read by Dr. A. F. Wells, Dr. H. Iipson and Dr. W. H. Taylor at a meeting held in the Royal Institution on November 10 to celebrate the fiftieth a nniversary of the discovery of X-rays. 